Dermatologische Zeitschrift. 1906;13:311-313

\title{
Rostocker Dissertationen
}

1. EtSUJiro, Ikeda (Japan), Hydroa vaccine formis (Bazin).

Kurze Mitteilung von 44 in der Literatur beschriebenen Fallen, denen Verfasser einen neuen aus der Rostoeker Universitätsklinik hinzufügt. Er betont den an sich harmlosen Charakter der Affektion, die jedoch zu einer schwerwiegenden werden kann durch die fortwährenden Rezidive, die Ent-stellung infolge der Narbenbildung, das gelegentliche Befallenwerden der vorderen Augenmedien. Man kann 2 Typen unterscheiden: Der bei weitem häufigste, der der Krankheit auch den Namen gegeben hat, ist der, bei dem die Effloreszenzen zur Zellen- und Narbenbildung tendieren, der weit seltenei e vesico-bullöse, bei der es meist nicht zur Narbenbildung kommt, scheint be-sonders dann aufzutreten, wenn die Affektion sich erst in späteren Jahren oinstellt. Gewöhnlich beginnt sie in der Kindheit und bessert sich oder verschwindet in einzelnen Fallen zwischen dem 25. und 30. Jahre Histo-logisch fand Verfasser entsprechend anderen Autoren Bildung einer multi-lokulären Blase im Rete, entstanden durch mechanische Auseinanderdrängung der Stachelzellen in und um die Blase, im Stratum Malpighi reichliche Leukozyten, im Corium Auflockerung des Bindegewebes und Dilatation der Gefässe, die von weissen und roten Blutkörperctien vollgepfropft und von einem dichten Mantel von Leukozyten umgeben sind.

2. Harm, Carl, Ein FalJ von Sklerodermia diffnsa et circumscrip ta mit Sklerodaktylie bei einem 9jährigen Mädchen.

Es handelt sich um ein bis dabin gesundes Kind, bei dem sich neben einer Stõrung der geistigen Entwicklung eine typische Sklerodermie ent-wickelt, die, an der unteren Extremität beginnend, im weiteren Verlauf das Bild der Sklerodaktylie, der zirkumskripten und der diffusen Form zeigt und nach einem interkurrenten Erysipel eine immerhin bemerkenswerte Besserung

312 Rostocker Dissertationen.

zeigt. Verfasser betont ausdriicklicli, dass er mit Wolters und Dickler einen wesentlichen Unterschied zwischen den genannten einzelnen Formen nicht anerkennt, weil sie sehr wohl

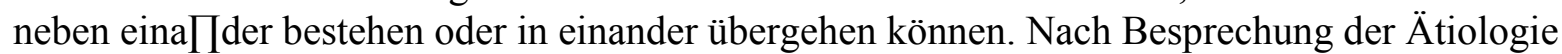
und Symptomatologie der Affektion geht er sehr ausfülirlich auf die von Unna und später von Zarubin erliobenen histologißchen Befunde ein, er selbst fand auch im mikroskopisclien Bilde keine Differenzen zwischen zirkumskripter und diffuser Form. 2 wohlgelungene Photographies sind der Arbeit beigegeben.

3. Grob $\theta 1$ ay, Joseph, Über Psoriasis nach Impfung.

In der Literatur sind mehrere Fälle von Auftreten einer Psoriasis nach Impfung beschrieben. Verfasser bespricht dieselben, soweit sie ihm zugänglich waren, und fügt zwei neue aus der Privatpraxis von Prof. Wolters bei. Beide Male trat die Affektion im unmittelbaren Anschluss an die 2. resp.

Impfung auf (5 resp. 8 Wochen später); beide Male lag hereditäre Be-lastung quoad Psoriasis vor. Da fast alle bisher beschriebenen ähnlichen Fälle mit Kälberlymphe geimpft waren, eine derartige Erkrankung bei den Tieren aber unbekannt ist und eine Übertragung durch die Impflanzette ausgeschlossen erscheint, so kann eine Übertragung der Krankheit durch den 
Impfakt nicht anerkannt werden. Dazu kommt, dass überhaupt die parasitäre Natur der Schuppenflechte und damit die Möglichkeit einer direkten Übertragung auf Andere auf sehr schwachen Füssen steht. Ein Krankheits-erreger ist bisher nicht einwandsfrei nachgewiesen. Verfasser nimmt an, dass die P. eine Stoffweohselanomalio sei, die durch ihr Bestehen einen derartigen Erregungszustand des Körpers und der Haut bedingt, dass iedes Trauma eine Hauteruption zur Folge hat. Auch die Impfung stellt ein solches Trauma dar, auf dessen Reiz die mit der supponierten Stoffwechselstörung (die ev. auch hereditär übertragen sein kann) behafteten Menschen mit einer Psoriasis-Eruption reagieren.

Kablitz, Richard, Ein Beitrag zur Frage der Epidermolysis bullosa traumatica (hereditaria et acquisita). Man unterscheidet klinisch 2 Formen der Epidermolysis, die eine mit, die andere ohne sekundäre Hautatrophie, Epidermiscysten und Nagelverände-rungen, erstere wurde hauptsächlich von englischen und französischen, letztere vorwiegend von deutschen $\Lambda$ utoren beschrieben. Beiden gemeinsam ist als wesentlichstes und hervorragendstes Symptom die Eutstehung der Blasen auf traumatischer Grundlage, während die früher als allgemeines Charakte-ristikum der Erkrankung aufgefasste Heredität heute nicht mehr fur so bedeut-sam angesehen wird, Nach genauer Würdigung der Literatur beschreibt Verf. selbst einen solchen, nicht hereditären Fall sehr genau, bei dem die Affektion erst im 60. Jahr begann und bei dem es ihm auch gelang, durch Quetschen der Haut Blasenbildung auszulösen. Über eigene histologische Befunde ver-fügt Verf. nicht, bespricht aber sehr ausfülirlich die Untersuchungen anderer und schliesst aus ihnen: Bei hereditären und nicht hereditären Fallen heilen die Bullae entweder ohno bleibende Veränderungen der Haut oder mit Hint $\beta r$-lassung von Narben und Milien, je nachdem sich die Blasen in höheren oder tieferen Schichten des Ret $\theta$ bilden. Narbenbildung und Nichtnarbenbildung, Vererbung und Nichtvßrerbung sind demnach nur zufällige Momente; nur die chronisch sich hinziehende Blasenbildung charakterisiert das Leiden. Zwei photographische Aufnahmen sind beigegeben.

5. $\quad$ Roth, Johs., Über subunguale Sarkome.

Verf. bespricht in dieser Arbeit die Tumoren sub ungue überhaupt, di · er in solche epithelíaler und solche bindegewebiger Abkunft einteilt. Von den ersteren wird der Clavus subungualis, die echte Verruca, das Carcinoma subunguale, von dem nur wenige Fälle bekannt sind, kurz beschrieben, von letzteren streift Verf. die Exostosen, die Enchondrome, das subunguale Papillom,

Rostocker Dissertationen. 313

einen Fall von Leiomyom und endlicli ausführlieh das Sarkom, von denen 14 Fãlle bekannt sind. Verf. hat selbst in Rostock einen einschlägigen Fall beobachtet, den er genau, auch in histologischer Beziehung, mitteilt, und be-spricht ausführlieh auf Grund der Literatur die Diagnose, Prognose, Sympto-matologie und mikroskopische Zusammensetzung der in Rede stehenden Tumoren.

6. Suzuki, Tokujirr, Stabsarzt der k. japanischen Marine, Über Syphilis im Altertum, speziell in China und Japan.

Die sehr ausführlieh gehaltene Arbeit gipfelt in folgenden Schlüssen: Es lässt sich weder aus den Schriften des Altertnms bis zum Jahre 1493, noch aus den Funden prähistorischer, krankhaft veränderter Knochen ein Anhaltspuokt dafür gewinnen, dass die Syphilis vor dem oben erwähnten Zeitpunkt in der alten Welt vorhanden gewesen ist. Es stützt daher dieser negative Befund die Tatsache, dass die Syphilis 1493 von den Leuten des Columbus eingeschleppt und von Spanien und Portugal aus, besonders durch den italienischen Feldzug, verbreitet wurde, 
unter andereni auch nach China und Japan, wo es vorher wohl lokale GeschlechtsleideD, nicht aber dasjenige Krankheitsbild gab, das wir heute als konstitutionelle Lues bezeichnen.

Art hur-Alexander- Berlin.

Buchanzeigen.

B10, Valdemar, Die Anvvendung des Lichtes in der Medizin. Mit besonderer Berücksichtigung von Professor Finsens Lebenswerk. Wiesbaden 1905. J. F. Bergmann.

Dem Leser dieser Zeitschrift bringt die Schrift von Bie schwerlich etwas Neues, denn sie ist eine zwar $\Lambda$ vissenschaftlich getreue, aber für das allgemeine Verständnis geschriebene $\Lambda$ Viedergabe der Finsenlehre, jedoch dient sie auch dem Erfahrenen zur Klärung der Ansichten, denn sie ist in konziser, alles Wesentliche berücksichtigender Fassung geschrieben. Die Wirkungen des Lichtes auf Haut, Stoffvvechsel und Blut, seine Fähigkeit einzudringen, $\mathrm{zu}$ irritieren und psychische Einflüsse hervorzubringen, seine hygienische und bakteriologische Bedeutung, sowie die Lichtbehandlung selbst werden in grossen Zügeu abgehandelt, alles in allem ein schönes und sachliches Denkmal für den unvergesslichen Begründer dieser ganzen Richtung. Auch als Unterlage für Vortrãge bei Einführung von Studierenden und Arzten in dies Gebiet, sowie zu populären A $\pi$ seinandersetzungen eignet sich dieser, man darf sagen, grössere Aufsatz. Lassar.

Bélot, J·, La radiotherapie, son application anx affections cutanées. Paris 1904. G. Steinheil. Bekanntlich zählt HerrJ. Bélot zu den besten Kennern und Vertretern der Rontgenologic nicht nur in seinem eigenen Vaterlande. Einen besseren Beweis als seine Radiotherapie der Hautkrankheiten könnte er hierfür nicht erbringen. Meisterhaft wie die kurze Geschichte wird die Theorie der X-Strahlen wiedergegeben. Selbst ein physikalisch mangelhaft Vorgebildeter kann hieraus die nötige Einführung erlernen, urn so mehr, als die exakte Methode der Radiotherapie bis in jede Einzelheit und mit vortrefflichen Illustrationen erklärt, alle einzelnen Punkte und Probleme erörtert werden. In Bezug auf die geschichtliche Entwicklung der Radiotherapie wird erwähnt, dass eigentlich erst seit dem Jahre 1901 die Franzosen angefangen haben, sich praktisch mit diesem Fache zu beschäftigen. Man kann dies nur billigen, da die vielfach hervorgerufeuen Radiodermatitiden, Verstümmelungen und Hautnekrosen wirklich zu äusserster Vorsicht gemahnt haben. Die empirische 